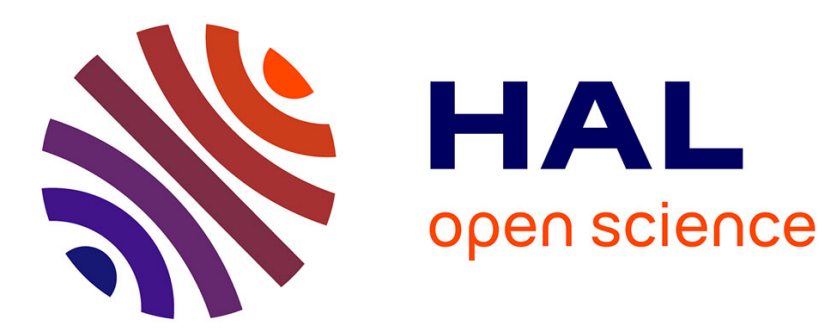

\title{
Innovation et réglementation
}

\author{
G. Letourneur
}

\section{To cite this version:}

G. Letourneur. Innovation et réglementation. Le Lait, 1984, 64 (638_639), pp.318-321. hal-00929013

\section{HAL Id: hal-00929013 \\ https://hal.science/hal-00929013}

Submitted on 1 Jan 1984

HAL is a multi-disciplinary open access archive for the deposit and dissemination of scientific research documents, whether they are published or not. The documents may come from teaching and research institutions in France or abroad, or from public or private research centers.
L'archive ouverte pluridisciplinaire HAL, est destinée au dépôt et à la diffusion de documents scientifiques de niveau recherche, publiés ou non, émanant des établissements d'enseignement et de recherche français ou étrangers, des laboratoires publics ou privés. 


\title{
CONCLUSIONS
}

\section{Innovation et réglementation}

\author{
par \\ G. LETOURNEUR
}

Tout au long de cette journée, chercheurs, pré-développeurs, équipementiers, nous ont précisé à quel niveau de connaissance et de maîtrise technique ils étaient parvenus en matière de technologies séparatives.

Ils nous ont laissé percevoir ce qu'en seront les développements à venir et ce que devraient en être les applications industrielles à l'horizon 90.

L'industrie agro-alimentaire est toute ouïe.

Elle sait que pour assurer son futur, il lui faut sans cesse :

- innover, développer, moderniser, diversifier.

Elle sait que ces objectifs forment un ensemble indissociable car :

- moderniser hors tout développement technologique de pointe se réduit pour elle à une simple opération de mise à niveau technique rapidement dépassée.

- diversifier ses produits hors toute innovation vraie se réduit à un banal rajeunissement qui ne résiste guère à l'usure du temps.

Elle sait que pour atteindre ces objectifs, seront nécessaires :

- De lourds et longs investissements de recherche.

Union Laitière Normande. 
- Une excellente coordination et complémentarité entre les divers partenaires.

- Une prompte et efficace exploitation des connaissances et résultats acquis car l'innovation ne vaut que par la rapidité de son passage dans la réalité des faits industriels.

Depuis quelques temps, il semble que nous ayons, tous ensemble, pris conscience de ces impératifs :

- A tous les niveaux de la filière, les efforts de recherche s'accentuent.

- Le dialogue cherche à se mieux établir et prend en compte les idées et les intérêts de chacun.

- Le délicat problème du transfert technologique semble devoir être progressivement mieux apprécié et compris de tous.

Tout cela est essentiel.

Tout cela risque, industriellement parlant, de n'être qu'investissements et efforts stériles si l'actuelle rigidité réglementaire demeure et si l'immobilisme continue à faire force de loi.

Pour mieux apprécier le danger, portons un regard sur le passé récent :

Voyons ce que, depuis 10 ou 15 ans, chercheurs et développeurs ont déjà mis à notre disposition même s'il demeure à faire et toujours mieux faire.

Voyons ce que les industriels ont, en France, été autorisés à entreprendre et développer.

Quelques exemples choisis au sein de la filière " lait " vaudront mieux qu'un long discours :

- L'ultrafiltration du lait... elle se heurte à des textes réglementaires rétrogrades, libellés voici 30 ou 50 ans, à une époque où techniques et produits ne pouvaient être définis bien sûr qu'en termes de matière grasse, lait écrémé, écrémage, égouttage, voire de moulage à la louche...

Résultat : la capacité qui devrait être nôtre de mieux utiliser et valoriser ces nutriments essentiels que sont les protéines laitières reste encore, à ce jour, partiellement lettre morte.

- L'ultrafiltration du lactosérum... elle se heurte aux imageries du passé qui font de cette matière première un quelconque et vil sous-produit collecté dans des conditions d'hygiène que l'on n'oserait décrire...

Résultat : le législateur, subjectivement, ne peut ni ne veut admettre que les composants unitaires de cette matière première puissent être considérés comme du bel et bon extrait sec laitier... notre capacité à concentrer et purifier, en particulier les protéines, que chimistes et nutritionnistes s'accordent à reconnaître comme 
de valeur biologique remarquable, connaît un développement lent et perturbé.

- La cristallisation et le fractionnement physique de la matière grasse butyrique se heurtent eux aussi aux appréciations purement subjectives du législateur qui ne veut, ni ne peut admettre que ces fractions puissent avoir droit au titre de nobles matières capables d'améliorer qualité et valeur nutritionnelle de produits tels que le beurre.

- L'hydrolyse du lactose attend depuis 10 ans que nutritionnistes et toxicologues aient enfin statué sur l'inocuité des produits qui en sont issus.

Et dans un tel contexte, vous continuez à préparer les voies de la troisième révolution industrielle... Bravo!

Mais, pour ne pas être déçus, pour qu'efforts et dynamismes mis à chercher, mettre au point, pré-développer ne soient autres choses que peines perdues... avouez qu'il est urgent que tous ensemble, nous tentions de faire évoluer la lourde machine administrative et les mentalités qui l'immobilisent plus encore.

La tâche sera rude.

On nous opposera que le temps mis à décider n'est que légitime prudence et qu'il se justifie par le souci de ne pas sacrifier santé et protection du consommateur à la folie innovatrice des transformateurs.

Si tel était bien le cas, nous ne pourrions qu'applaudir car aussi surprenant que cela puisse être, nous sommes tous attachés à la satisfaction optimale de notre clientèle.

Malheureusement, ce temps qui passe n'est actuellement que le résultat d'atermoiements inefficaces, de lenteurs accumulées, d'attitudes passéistes et frileuses...

Il n'apporte aucune sécurité vraie.

Pour espérer mettre un terme à cette situation, il nous faut faire passer un petit nombre de messages précis, il nous faut ne pas hésiter à balayer devant notre porte, il nous faut proposer des moyens et façons de faire porteurs d'efficacité nouvelle.

Parmi ces attitudes, procédures et moyens, nous citerons :

- L'abandon par les transformateurs de l'autoprotectionnisme par voie règlementaire dont il est agréable de savourer les délices dans un marché peu concurrentiel, mais dont on oublie qu'il est source de stagnation dans un marché ouvert.

- La nécessité pour tous les partenaires que nous sommes de nous attacher, très tôt, à faire cheminer de paire les recherches techniques et les réflexions d'ordre règlementaire de façon à ce qu'évolutions et adaptations puissent, le moment venu, être adoptées sans excessifs retards. 
- L'urgence qu'il y aurait à ne plus juger, définir et autoriser les techniques et produits par références aux us et coutumes du passé, mais à les évaluer en termes de service, sécurité, valeur nutritionnelle, apportés et garantis au consommateur final.

- L'impérieux et urgent besoin de, pour cela, créer en France un véritable institut de l'alimentation et de la nutrition. Cet institut devrait rassembler toutes les compétences et moyens dont, en ces domaines, notre pays, d'ores et déjà, dispose.

Il devrait être investi d'autorité scientifique et de responsabilités vraies.

Ses devoirs seraient d'efficacement et objectivement répondre à toute question posée par l'homologation d'une technologie nouvelle, l'agrément d'une matière première ou d'un produit fini nouveau.

En l'absence d'un tel institut opérationnel d'expertise et d'arbitrage, l'industrie agro-alimentaire continuera à voir tous ces procédés porteurs d'avenir dont vous nous avez encore aujourd'hui entretenus.

- N'être reçus et traités qu'en termes subjectifs et passionnels.

- Ne prendre leurs dimensions industrielles vraies qu'après 10 ou 15 ans de vains atermoiements. Or à notre époque, ce laps de temps équivaut à une génération technologique, à une génération produit...

N'a-t-on pas là une explication du cruel retard que l'on s'ingénie à ne reprocher qu'aux seuls entrepreneurs ?

- Ne continuer à être malheureusement mis trop souvent à notre disposition qu'après qu'ils aient été expérimentés et fiabilisés à l'extérieur de nos frontières, donc sous forme de techniques et produits étrangers...

N'a-t-on pas là une explication de notre dépendance que l'on s'ingénie à ne reprocher qu'aux seuls équipementiers et transformateurs?

Nous ne voulons pas croire que ce soient là les résultats et objectifs que Pouvoirs Publics et entreprises espèrent et se fixent lorsqu'ils décident ensemble de mieux investir et collaborer dans la recherche et le développement.

Nous ne voulons pas croire que par le biais de certaines de leurs composantes, les Pouvoirs Publics chercheront à dynamiser ces activités essentielles tandis que d'autres n'auront de cesse d'en stériliser les fruits.

Nous voulons croire que dans le respect bien compris des intérêts de l'ensemble des partenaires socio-économiques (consommateurs, transformateurs, équipementiers, chercheurs), il sera rapidement possible de mettre en œuvre de vraies et réelles réformes pour la sécurité et la satisfaction de tous...

Et qu'il ne nous faudra pas attendre le 29 février 1988. 